\title{
A function for characterizing complete kinetostatic behaviors of compliant bistable mechanisms
}

\author{
G. Li and G. Chen \\ School of Mechatronics, Xidian University, Xi' an, Shaanxi 710071, China \\ Correspondence to: G. Chen (guimin.chen@gmail.com)
}

Received: 16 July 2014 - Revised: 17 November 2014 - Accepted: 20 November 2014 - Published: 12 December 2014

\begin{abstract}
In this paper, a straightforward and accurate numerical modeling (a rational function called "tri-root bistable function") are proposed to represent the complete nonlinear bistable force-displacement characteristics. The rational function has a cubic polynomial numerator and quadratic polynomial denominator. With three different kinds of compliant bistable mechanisms, the tri-root bistable function is proved effective and accurate, and that it is capable of capturing the key features of a bistable kinetostatic curve accurately with fewer parameters. Then, for the classic fully-compliant bistable mechanism, six closed-form equations are presented and used to describe the relationships between the tri-root bistable function parameters and the mechanism's design parameters, which are achieved using a multi-variable nonlinear regression. The regression analysis is validated by nonlinear finite element analysis. Finally, a fully-compliant statically balanced mechanism consisting of three different classic fully-compliant bistable mechanisms is illustrated to show the capability of the proposed method in designing compliant multi-stable mechanisms.
\end{abstract}

\section{Introduction}

A compliant bistable mechanism is a device that can maintain two distinct positions without power input (Howell, 2001). A multitude of different compliant bistable mechanisms has been proposed and studied (Su and McCarthy, 2007; Jensen et al., 2001; Hansen et al., 2007; Jensen and Howell, 2004; Masters and Howell, 2003; Wilcox and Howell, 2005; Sönmez and Tutum, 2008; Jensen et al., 1999; Jensen and Howell, 2003). These mechanisms have many potential applications such as switches (Oberhammer et al., 2006; Hoffmann et al., 1999), closures, relays (Charlot et al., 2008), reconfigurable robots (Hafez et al., 2003), and landing gear mechanisms (PuchetaMMT and Cardona, 2010). Due to their negative-stiffness behaviors around the unstable equilibrium positions (a bistable mechanism has two stable equilibrium positions and an unstable equilibrium position), they have been used as negative stiffness building blocks for achieving static balancing in compliant mechanisms (Tolou et al., 2011; Chen and Zhang, 2011). Bistable mechanisms of different types have also been used as fundamental building blocks for synthesis of multistable mechanisms that have more than two stable positions (Chen et al., 2009b, a; Oh and Kota,
2009; Chen et al., 2011b; Chen and Du, 2013; Halverson et al., 2010; Pendleton and Jensen, 2007). Andò et al. (2012) also utilized a bistable mechanism for energy harvesting. The wide use of bistable mechanisms motivates the development of an approach for accurately modeling their kinetostatics (i.e., the load-displacement relationship).

However, the nonlinearity associated with the bistable behavior often complicates the modeling. The pseudo-rigidbody method (PRBM) (Howell, 2001), which approximates the nonlinear deflection as motion of rigid links, is particularly useful in the early design phases of bistable mechanisms, but inaccurate for the later design phases. Currently nonlinear finite element analysis (NFEA) is still an important tool for obtaining accurate kinetostatic behavior of bistable mechanisms during the late design phases.

In this paper, we propose a rational function called "triroot bistable function" for the purpose of easily and explicitly representing the complete kinetostatic behaviors of bistable mechanisms. This function provides bistable mechanism designers an explicit and easy way to represent their design requirements for bistable behaviors. The tri-root bistable function has a 3rd degree polynomial numerator and a 2nd de- 


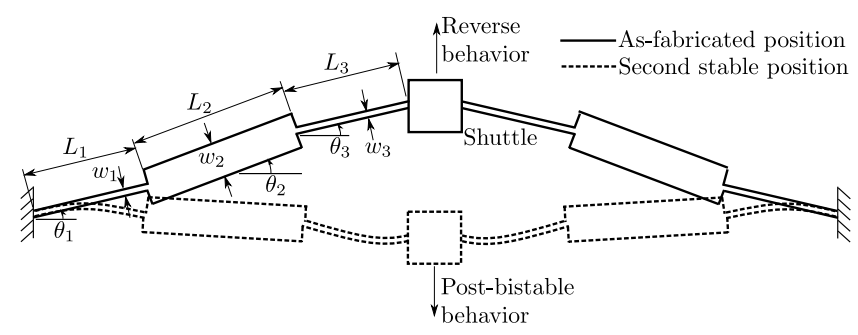

Figure 1. A classic fully-compliant bistable mechanism.

gree polynomial denominator, and is capable of capturing the key features of a complete bistable kinetostatic curve (including the external kinetostatic behaviors, Chen et al., 2011b) accurately with fewer parameters. If the key points of a kinetostatic behavior are given as the design requirements of a bistable mechanism, the tri-root bistable function enables the construction of the whole bistable curve. Using the constructed function as the target bistable curve, an optimization process can be employed to search for a desired bistable mechanism design (Chen and Du, 2013). Moreover, for a given type of bistable mechanism, a regression analysis from available NFEA and/or experimental results can identify the relationships between the tri-root bistable function parameters and the mechanism's design parameters (including both the geometric and the physical parameters). Once the relationships are known for a specific type of bistable mechanisms the design of future mechanisms of this type is greatly simplified.

The rest of this paper is organized as follows: Sect. 2 reviews different ways for describing the complete bistable kinetostatic behaviors and presents this new rational function. Section 3 illustrates how accurate the rational function is in representing the complete bistable kinetostatic behaviors using three different kinds of compliant bistable mechanisms. Section 4 presents the closed-form equations of the kinetostatic behavior of a classic fully-compliant bistable mechanism, which are achieved using a multi-variable nonlinear regression (a mathematical optimization and analysis software called 1stOpt is used). In Sect. 5, a fully-compliant statically balanced mechanism consisting of three different classic fully-compliant bistable mechanisms is illustrated to validate the accuracy of the six closed-form equations. The concluding remarks are made in the last section.

\section{Mathematical representations of bistable kinetostatic behaviors}

In this section, we will take the fully-compliant bistable mechanism (Jensen et al., 2001) shown in Fig. 1 as an example to demonstrate the representation of bistable behaviors. Figure 2 plots the kinetostatic behavior curve of a bistable mechanism whose design parameters are listed in Table 1, which are achieved using nonlinear finite element analysis

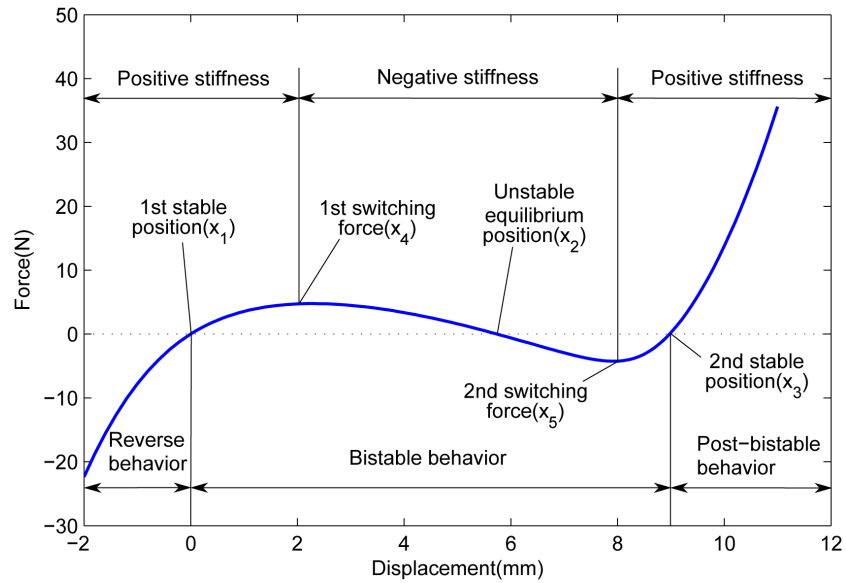

Figure 2. The complete kinetostatic behavior curve of a bistable mechanism.

(NFEA). The whole curve can be divided into three sections: the bistable behavior section, the reverse behavior section (Sönmez and Tutum, 2008) and the post-bistable behavior (Wilcox and Howell, 2005) section. We denote the whole force-deflection characteristics as

$F=F(x)$

where $x$ is the travel distance of the shuttle from its first stable equilibrium position (i.e., the as-fabricated position) along the $y$ axis. As illustrated in Fig. 2, the function should have three roots, i.e., $x_{1}, x_{2}$ and $x_{3}$, where $x_{1}$ and $x_{3}$ correspond to the two stable equilibrium positions while $x_{2}$ corresponds to the unstable equilibrium potion. Moreover, $x_{4}$ and $x_{5}$ correspond to the two switching positions named by Hansen et al. (2007).

In order to model this highly nonlinear behaviors of this type of mechanism, Oh and Kota (2009) employed a piecewise third-order function to approximate the bistable behaviors:

$$
F_{1}(x)= \begin{cases}f_{1}(x) & x_{1} \leq x<x_{4} \\ f_{2}(x) & x_{4} \leq x<x_{5} \\ f_{3}(x) & x_{5} \leq x \leq x_{3}\end{cases}
$$

satisfying

$$
\begin{aligned}
& F_{1}\left(x_{1}\right)=F_{1}\left(x_{3}\right)=F_{1}(0)=0 \\
& \left.\frac{\mathrm{d} F_{1}}{\mathrm{~d} x}\right|_{x=x_{4}}=\left.\frac{\mathrm{d} F_{1}}{\mathrm{~d} x}\right|_{x=x_{5}}=0 \\
& f_{1}\left(x_{4}\right)=f_{2}\left(x_{4}\right), f_{2}\left(x_{5}\right)=f_{3}\left(x_{5}\right)
\end{aligned}
$$

It was suggested that subfunctions $f_{1}(x)$ and $f_{2}(x)$ were second-order polynomials, and $f_{3}(x)$ was a third-order polynomial. As shown in Fig. 3, the function $F_{1}(x)$ can be yielded 
Table 1. Design parameters of a classic fully-compliant bistable mechanism. $H$ is the out-of-plane thickness of the mechanism, $E$ is the Young's modulus of polypropylene.

\begin{tabular}{cccccccccccc}
\hline Parameter & $E$ & $H$ & $L_{1}$ & $\theta_{1}$ & $w_{1}$ & $L_{2}$ & $\theta_{2}$ & $w_{2}$ & $L_{3}$ & $\theta_{3}$ & $w_{3}$ \\
\hline Value & $1.4 \times 10^{9} \mathrm{~Pa}$ & $6 \mathrm{~mm}$ & $8 \mathrm{~mm}$ & $0^{\circ}$ & $0.8 \mathrm{~mm}$ & $20 \mathrm{~mm}$ & $12^{\circ}$ & $3 \mathrm{~mm}$ & $8 \mathrm{~mm}$ & $0^{\circ}$ & $0.8 \mathrm{~mm}$ \\
\hline
\end{tabular}

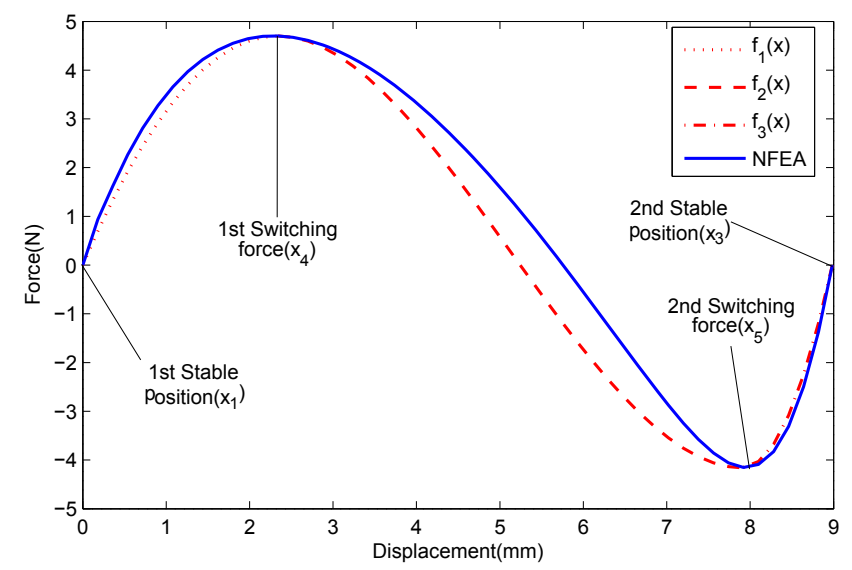

Figure 3. The comparision between the third-order polynomial and NFEA.

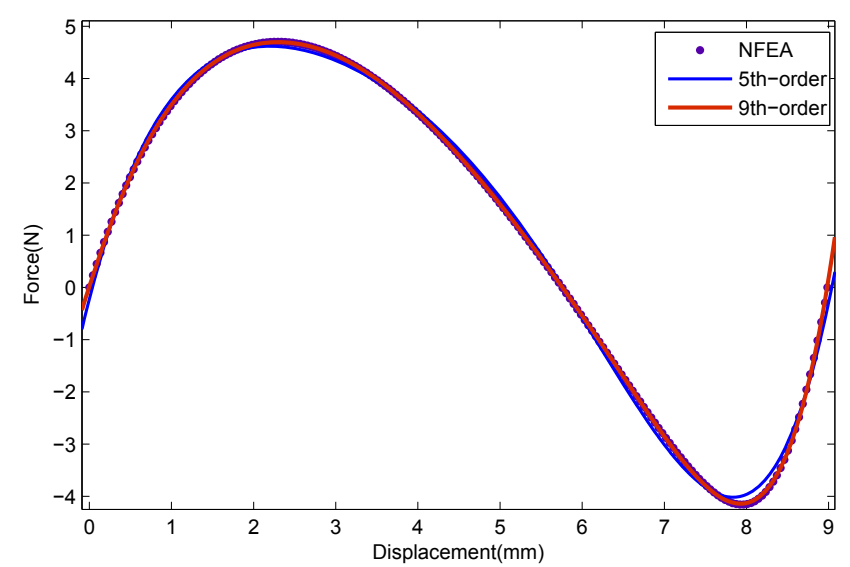

Figure 4. The comparision among the fifth, ninth-order polynomials and NFEA.

as:

$$
\begin{array}{cc}
F_{1}(x)= & \\
f_{1}(x)=-0.8588 x^{2}+4.0191 x & 0 \leq x<2.34 \\
f_{2}(x)=0.1019 x^{3}-1.5686 x^{2}+5.6666 x-1.2747 & 2.34 \leq x<7.92 \\
f_{3}(x)=3.6603 x^{2}-57.9791 x+225.446 & 7.92 \leq x \leq 8.985
\end{array}
$$

As shown in Fig. 3, Eq. (6) satisfies the key points of the bistable behavior accurately, but cannot describe the whole bistable behavior well.

Wang et al. (2009) used a ninth-order polynomial to model the nonlinear spring stiffness of the mechanism as

$F_{2}(x)=\sum_{i=0}^{9} k_{i} x^{i}$

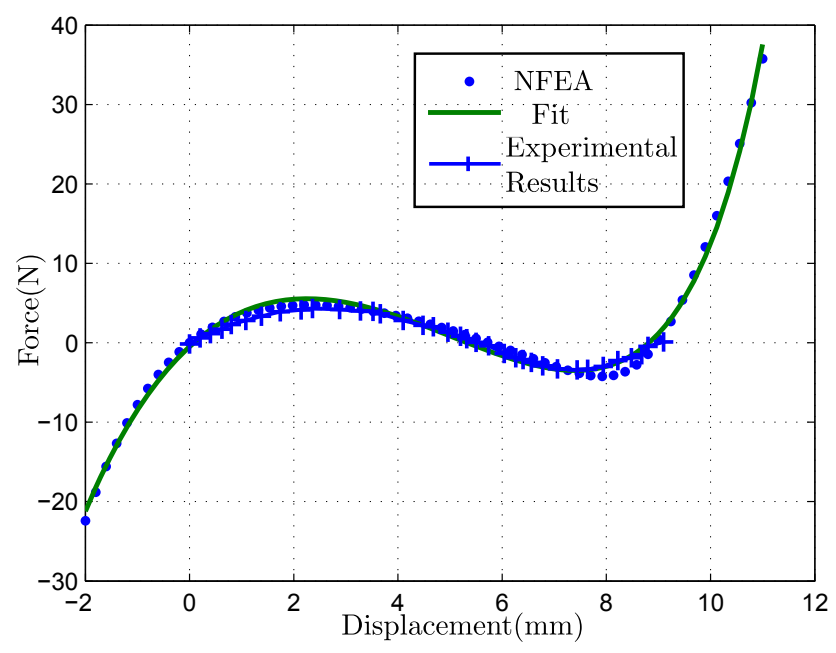

Figure 5. The complete bistable kinetostatic behaviors of the classic fully-compliant bistable mechanism.

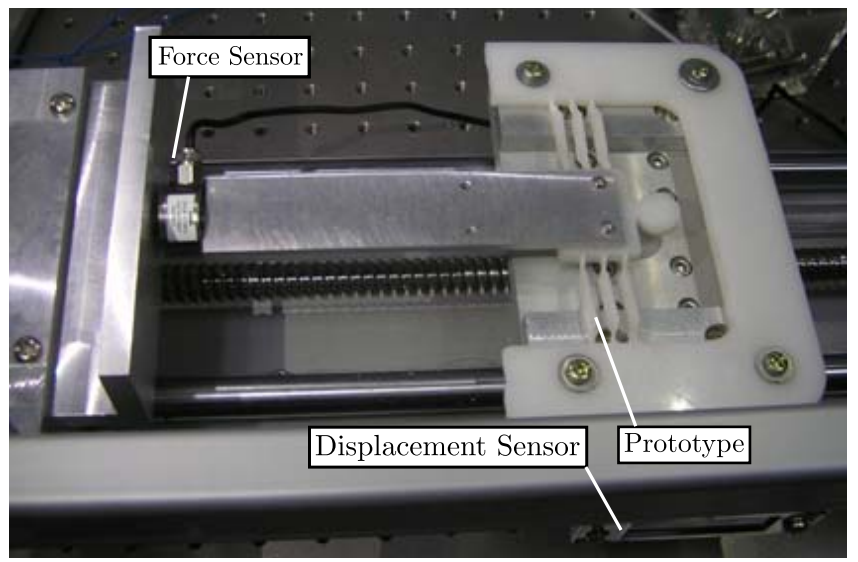

Figure 6. Experimental setup.

Third, fifth, seventh, eighth and ninth-order polynomials have been used to model the highly nonlinear forcedisplacement curve. However, the curves generated by the polynomials with order less than nine do not fit the forcedisplacement curve well, as shown in Fig. 4, and the expression can not capture the bistable behaviors directly.

To address these problems, this paper proposes a continuous rational function called "tri-root bistable function" to accurately describe the complete kinetostatic behaviors of the bistable mechanisms with fewer parameters. Considering that the bistable curve contains three zeros, the tri- 

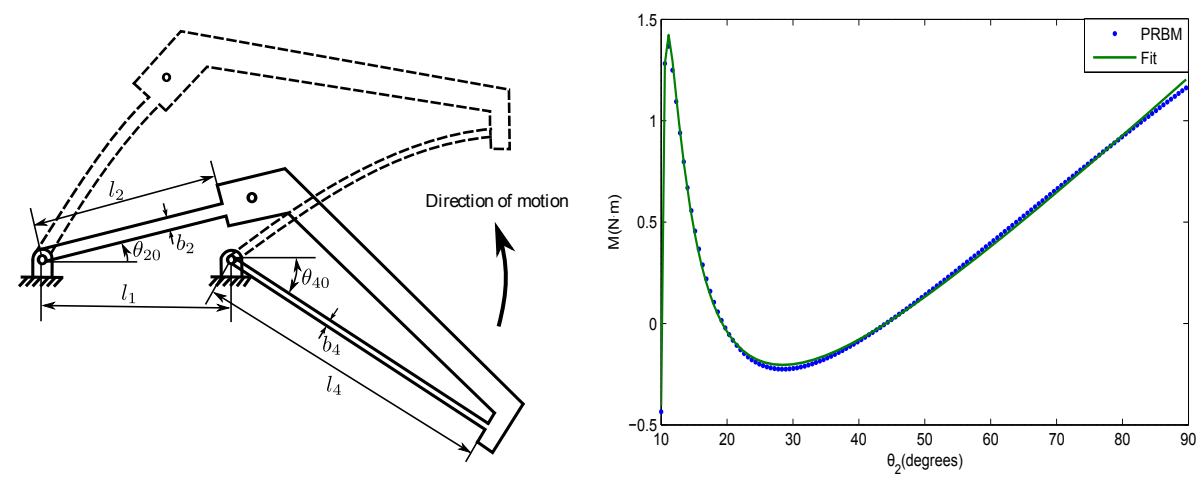

Figure 7. The structure of a Young bistable mechanism and its bistable kinetostatic behaviors.

Table 2. Design parameters of a Young bistable mechanism. $H$ is the out-of-plane thickness of the mechanism, $E$ is the Young's modulus of polypropylene.

\begin{tabular}{cccccccccc}
\hline Parameter & $E$ & $H$ & $l_{1}$ & $\theta_{20}$ & $l_{2}$ & $\theta_{40}$ & $l_{4}$ & $b_{2}$ & $b_{4}$ \\
\hline Value & $1.4 \times 10^{9} \mathrm{~Pa}$ & $6 \mathrm{~mm}$ & $128 \mathrm{~mm}$ & $10^{\circ}$ & $110 \mathrm{~mm}$ & $40^{\circ}$ & $174 \mathrm{~mm}$ & $6 \mathrm{~mm}$ & $3 \mathrm{~mm}$ \\
\hline
\end{tabular}

root bistable function has a cubic polynomial numerator and quadratic polynomial denominator (we empirically chose the denominator based on the testing results among different functions) and is expressed as

$$
\begin{aligned}
F_{3}(x) & =\frac{p_{1}\left(x-x_{1}\right)\left(x-x_{2}\right)\left(x-x_{3}\right)}{x^{2}+q_{1} x+q_{2}} \\
& =\frac{p_{1} x^{3}+p_{2} x^{2}+p_{3} x+p_{4}}{x^{2}+q_{1} x+q_{2}}
\end{aligned}
$$

where, $\left(4 q_{2}-q_{1}^{2}\right)>0$ (i.e., $\left.\left(x^{2}+q_{1} x+q_{2}\right)>0\right), p_{1}>0$ and $x_{1}<x_{2}<x_{3}\left(x_{1}, x_{2}\right.$ and $x_{3}$ correspond to the three zeros of $F_{3}$ ). According to Vieta's formulas, the relationships between the three zeros and parameters $p_{i}(i=1,2,3,4)$ are given as:

$$
\left.\begin{array}{c}
x_{1}+x_{2}+x_{3}=-\frac{p_{2}}{p_{1}} \\
x_{1} x_{2}+x_{2} x_{3}+x_{1} x_{3}=\frac{p_{3}}{p_{1}} \\
x_{1} x_{2} x_{3}=-\frac{p_{4}}{p_{1}}
\end{array}\right\}
$$

For the five key points shown in Fig. 2, we have

$$
\left.\begin{array}{l}
F_{3}\left(x_{1}\right)=\frac{p_{1} x_{1}^{3}+p_{2} x_{1}^{2}+p_{3} x_{1}+p_{4}}{x_{1}^{2}+q_{1} x_{1}+q_{2}}=p_{1} x_{1}^{3}+p_{2} x_{1}^{2}+p_{3} x_{1}+p_{4}=0 \\
F_{3}\left(x_{2}\right)=\frac{p_{1} x_{2}^{3}+p_{2} x_{2}^{2}+p_{3} x_{2}+p_{4}}{x_{2}^{2}+q_{1} x_{2}+q_{2}}=p_{1} x_{2}^{3}+p_{2} x_{2}^{2}+p_{3} x_{2}+p_{4}=0 \\
F_{3}\left(x_{3}\right)=\frac{p_{1} x_{3}^{3}+p_{2} x_{3}^{2}+p_{3} x_{3}+p_{4}}{x_{3}^{2}+q_{1} x_{3}+q_{2}}=p_{1} x_{3}^{3}+p_{2} x_{3}^{2}+p_{3} x_{3}+p_{4}=0
\end{array}\right\}
$$

Taking the first derivative of $F_{3}$ with respect to $x$ yields the stiffness of the compliant bistable mechanism:

$F_{3}^{\prime}(x)=$

$\frac{\left(3 p_{1} x^{2}+2 p_{2} x+p_{3}\right)\left(x^{2}+q_{1} x+q_{2}\right)-\left(2 x+q_{1}\right)\left(p_{1} x^{3}+p_{2} x^{2}+p_{3} x+p_{4}\right)}{\left(x^{2}+q_{1} x+q_{2}\right)^{2}}$

thus we have

$F_{3}^{\prime}\left(x_{4}\right)=$

$\frac{\left(3 p_{1} x_{4}^{2}+2 p_{2} x_{4}+p_{3}\right)\left(x_{4}^{2}+q_{1} x_{4}+q_{2}\right)-\left(2 x_{4}+q_{1}\right)\left(p_{1} x_{4}^{3}+p_{2} x_{4}^{2}+p_{3} x_{4}+p_{4}\right)}{\left(x_{3}^{2}+q_{1} x_{4}+q_{2}\right)^{2}}=0$

$F_{3}^{\prime}\left(x_{5}\right)=$

$\frac{\left(3 p_{1} x_{5}^{2}+2 p_{2} x_{5}+p_{3}\right)\left(x_{5}^{2}+q_{1} x_{5}+q_{2}\right)-\left(2 x_{5}+q_{1}\right)\left(p_{1} x_{5}^{3}+p_{2} x_{5}^{2}+p_{3} x_{5}+p_{4}\right)}{\left(x_{5}^{2}+q_{1} x_{5}+q_{2}\right)^{2}}=0$

and

$$
\left.\begin{array}{l}
F_{3}\left(x_{4}\right)=\frac{p_{1} x_{4}^{3}+p_{2} x_{4}^{2}+p_{3} x_{4}+p_{4}}{x_{4}^{2}+q_{1} x_{4}+q_{2}}=F_{\max } \\
F_{3}\left(x_{5}\right)=\frac{p_{1} x_{5}^{3}+p_{2} x_{5}^{2}+p_{3} x_{5}+p_{4}}{x_{5}^{2}+q_{1} x_{5}+q_{2}}=F_{\min }
\end{array}\right\}
$$

At the first and second switching positions ( $x_{4}$ and $x_{5}$ ), the stiffnesses of the mechanism are equal to zero.

Furthermore, the total stain energy stored in a deflected bistable mechanism can be obtained by integrating the triroot bistable function: 


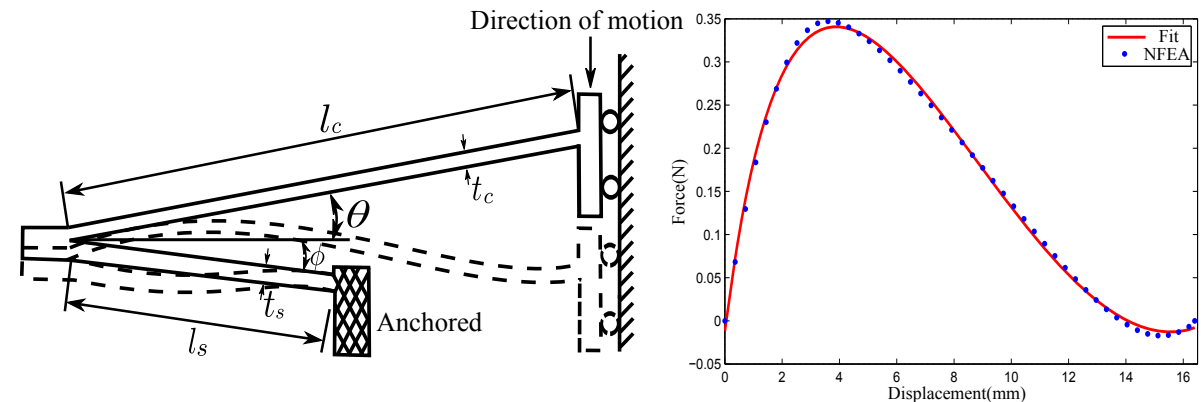

Figure 8. The structure of the fully compliant tensural bistable mechanism and its bistable kinetostatic behaviors.

Table 3. Design parameters of the fully compliant tensural bistable mechanism (FTBM). $H$ is the out-of-plane thickness of the mechanism, $E$ is the Young's modulus of polypropylene.

\begin{tabular}{ccccccccc}
\hline Parameter & $E$ & $H$ & $l_{c}$ & $\theta$ & $t_{c}$ & $l_{s}$ & $\phi$ & $t_{s}$ \\
\hline Value & $1.4 \times 10^{9} \mathrm{~Pa}$ & $6 \mathrm{~mm}$ & $120 \mathrm{~mm}$ & $5.5^{\circ}$ & $3.5 \mathrm{~mm}$ & $25 \mathrm{~mm}$ & $13^{\circ}$ & $1.5 \mathrm{~mm}$ \\
\hline
\end{tabular}

$$
\begin{aligned}
& V(x)=\int_{x_{1}}^{x} F_{3}(x) \mathrm{d} x=\int_{x_{1}}^{x} \frac{p_{1} x^{3}+p_{2} x^{2}+p_{3} x+p_{4}}{x^{2}+q_{1} x+q_{2}} \mathrm{~d} x \\
& =\frac{2 p_{4}-p_{3} q_{1}+p_{2} q_{1}^{2}-p_{1} q_{1}^{3}-2 p_{2} q_{2}+3 p_{1} q_{1} q_{2}}{\sqrt{-q_{1}^{2}+4 q_{2}}} \arctan \left(\frac{2 x+q_{1}}{\sqrt{-q_{1}^{2}+4 q_{2}}}\right) \\
& +\frac{1}{2}\left[p_{1} x^{2}+2 p_{2} x-2 p_{1} q_{1} x+\left(p_{3}-p_{2} q_{1}+p_{1} q_{1}^{2}-p_{1} q_{2}\right) \ln \left(x^{2}+q_{1} x+q_{2}\right)\right]
\end{aligned}
$$

At the first and second stable equilibrium positions $\left(x=x_{1}\right.$ and $x=x_{3}$ ), the total energies are minimum.

For a compliant bistable mechanism, if the five key points of the kinetostatic behavior curve are given as the design requirements (i.e. the five key points are known), the whole bistable curve can be plotted by the tri-root bistable function, which can be achieved easily by solving Eqs. (10), (12), (13) and (14) simultaneously. Because there are six parameters in Eq. (8), only six equations are required among these seven equations. In this paper, we take Eqs. (10), (13) and (14) to obtain the solutions for the six parameters. In addition, when the fitting curves passing through the origin (i.e., $F\left(x_{1}\right)=0$ when $x_{1}=0$ ), the accuracy of the regression analysis may be ruined (Eisenhauer, 2003), which can be avoided by translating the initial position to $(-1,0)$.

Conversely, for a given compliant bistable mechanism, the bistable kinetostatic behaviors can be captured easily and explicitly by the tri-root bistable function instead of the PRBM and NFEA. This requires us to find the relationships among the five key points and the design parameters firstly, which will be discussed in the following part of this paper.

\section{Fitting results of different types of bistable mechanisms}

In this section, three different types of compliant bistable mechanisms are utilized to illustrate the use of the tri-root bistable function in representing kinetostatic behaviors of the bistable mechanisms.

\subsection{Classic fully-compliant bistable mechanism}

The classic fully-compliant bistable mechanism introduced in Sect. 2 is chose as an illustration, whose configuration is shown in Fig. 1 and design parameters are listed in Table 1. Then, we use the tri-root bistable function (Eq. 8) to model its complete kinetostatic behaviors (including the reverse behavior (Sönmez and Tutum, 2008), the bistable behavior and the post-bistable behavior (Wilcox and Howell, 2005)), which are compared with nonlinear finite element analysis (NFEA). As shown in Fig. 5, the function $F_{L}(x)$ is yielded as:

$F_{L}(x)=\frac{-6.8286 x^{3}+99.9394 x^{2}-345.6224 x-14.322}{x^{2}-2.0921 x-77.2396}$

Figure 5 and Eq. (16) show that, the tri-root bistable function (Eq. 8) can capture the complete kinetostatic behaviors (including reverse behavior and post-bistable behavior) of the classic fully-compliant bistable mechanism with fewer parameters. The accuracy of the function can be improved when the kinetostatic behaviors don't include the reverse and post-bistable behaviors. Fitting through the origin may reduce the accuracy of the fitting results (Eisenhauer, 2003), so we chose $(-1,0)$ as the initial positions of the bistable curves to improve the fitting quality. The fitting results then were translated along the $x$ axis so that they pass through the origin for the purpose of comparing them to the NFEA and experimental results. 


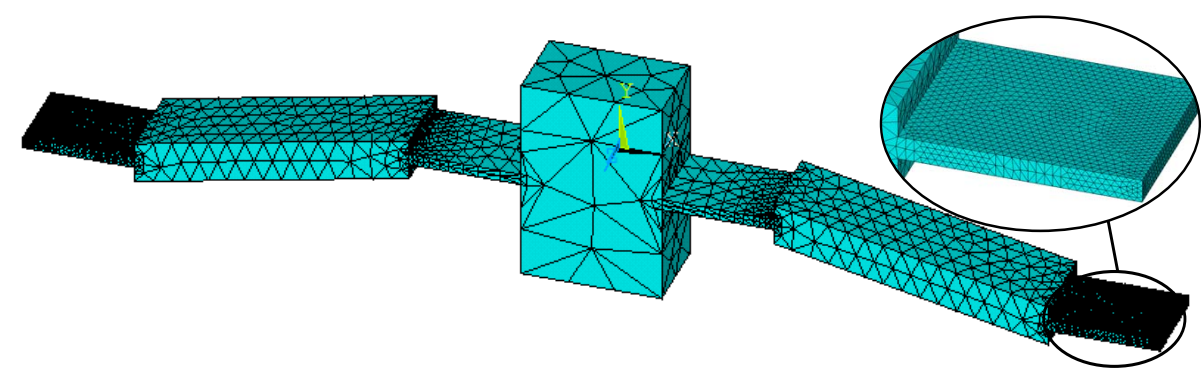

Figure 9. The finite element model for the classic fully-compliant bistable mechanism.
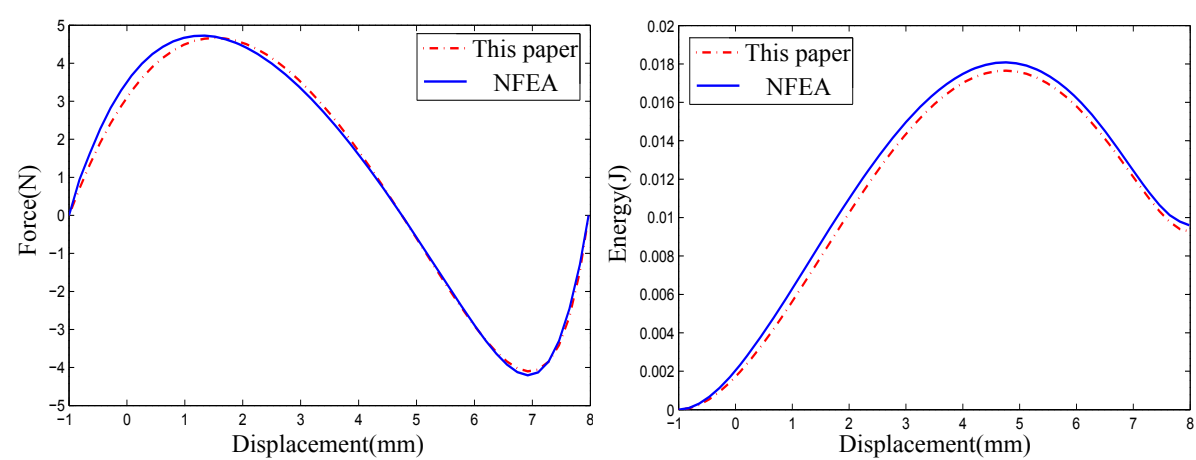

Figure 10. The comparison of the bistable kinetostatic behaviors achieved using NFEA and the tri-root bistable function.

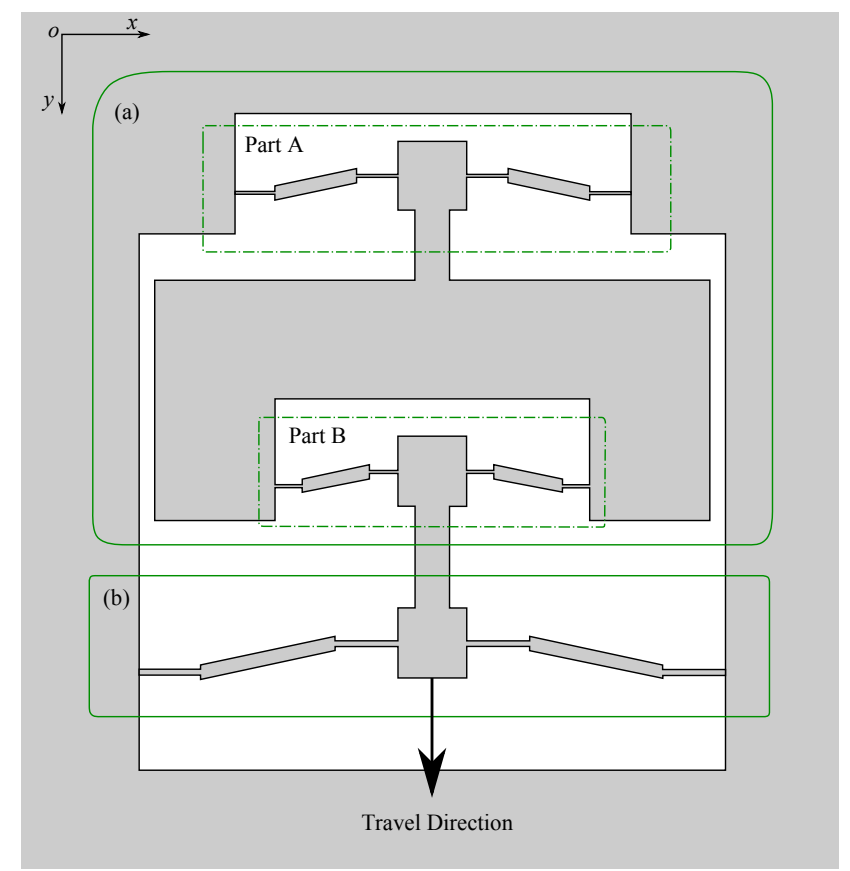

Figure 11. Structure of a fully-compliant statically-balanced mechanism consisting of three classic fully-compliant bistable mechanisms. (a) The compliant tristable mechanism consisting of two fully bistable mechanisms (i.e., Part A and Part B), and (b) the balancing bistable mechanism.
To examine the accuracy of the NFEA, a prototype of the mechanism was fabricated and measured, as shown in Fig. 6. The measured results are also plotted in Fig. 5. The measured three equilibrium positions are in good agreement with the FEA results. The measured two critical forces (the minimum forces required to transit full compliant bistable mechanism between its two stable positions) are slightly smaller than the NFEA predictions, which may be attributed to the machining errors of the prototype.

\subsection{Young bistable mechanisms}

With two articulated joints, the Young bistable mechanisms (Jensen et al., 1999) have double links: one of the links rigid (the ground link) and the other contains two flexible segments. Figure 7 shows the configuration of a Young bistable mechanism, which has two compliant links connected to the same coupler and the substrate. The design parameters are listed in Table 2.

Then, we use the tri-root bistable function (Eq. 8) to model its bistable kinetostatic behaviors and compare with the PRB method, as shown in Fig. 7. The function $M_{Y}(\theta)$ is obtained as:

$M_{Y}(\theta)=\frac{0.03108 \theta^{3}-2.282 \theta^{2}+46.25 \theta-266}{\theta^{2}-16.94 \theta+70.93}$

where, $\theta$ is the PRBM angle of $l_{2}$.

Figure 7 shows that, the tri-root bistable function (Eq. 8) is capable of capturing the key features of the kinetostatic 
Table 4. Design parameters of the fully-compliant staticallybalanced mechanism. $H$ is the out-of-plane thickness of the mechanism, $E$ is the Young's modulus of polypropylene.

\begin{tabular}{lrrr}
\hline Parameter & $\begin{array}{r}\text { Part A of } \\
\text { Tristable } \\
\text { Mechanism }\end{array}$ & $\begin{array}{r}\text { Part B of } \\
\text { Tristable } \\
\text { Mechanism }\end{array}$ & $\begin{array}{r}\text { Balancing } \\
\text { Bistable } \\
\text { Mechanism }\end{array}$ \\
\hline$E$ & $1.4 \times 10^{9} \mathrm{~Pa}$ & $1.4 \times 10^{9} \mathrm{~Pa}$ & $1.4 \times 10^{9} \mathrm{~Pa}$ \\
$H$ & $6 \mathrm{~mm}$ & $6 \mathrm{~mm}$ & $6 \mathrm{~mm}$ \\
$L_{1}$ & $12 \mathrm{~mm}$ & $8 \mathrm{~mm}$ & $7.95 \mathrm{~mm}$ \\
$\theta_{1}$ & $0^{\circ}$ & $0^{\circ}$ & $0^{\circ}$ \\
$w_{1}$ & $0.6 \mathrm{~mm}$ & $0.8 \mathrm{~mm}$ & $0.8 \mathrm{~mm}$ \\
$L_{2}$ & $24 \mathrm{~mm}$ & $20 \mathrm{~mm}$ & $38.6 \mathrm{~mm}$ \\
$\theta_{2}$ & $12^{\circ}$ & $12^{\circ}$ & $12.2^{\circ}$ \\
$w_{2}$ & $4 \mathrm{~mm}$ & $4 \mathrm{~mm}$ & $3 \mathrm{~mm}$ \\
$L_{3}$ & $12 \mathrm{~mm}$ & $8 \mathrm{~mm}$ & $1.1 \mathrm{~mm}$ \\
$\theta_{3}$ & $0^{\circ}$ & $0^{\circ}$ & $0^{\circ}$ \\
$w_{3}$ & $0.6 \mathrm{~mm}$ & $0.8 \mathrm{~mm}$ & $0.8 \mathrm{~mm}$ \\
\hline
\end{tabular}

behaviors of the Young mechanisms accurately with fewer parameters.

\subsection{Fully compliant tensural bistable mechanisms (FTBM)}

Figure 8 shows the configuration of a fully compliant tensural bistable mechanism (FTBM) (Wilcox and Howell, 2005), which consists of at least one primary segment which undergoes deformation and stores strain energy, and subjected to tension or bending loads (linear bistable micromechanisms that undergo tension loads, in addition to the bending loads present, through their range of motion). The design parameters are listed in Table 3.

Then, we use the tri-root bistable function (Eq. 8) to model its bistable kinetostatic behaviors and compare with the nonlinear finite element analysis (NFEA), as shown in Fig. 8. The function $F_{T}(x)$ is

$F_{T}(x)=\frac{-0.22 x^{3}+6.8124 x^{2}-52.4386 x+2.3393}{x^{2}-35.7666 x-203.164}$

As shown in Fig. 8, the tri-root bistable function (Eq. 8) is capable of capturing the key features of the bistable kinetostatic behaviors of the fully compliant tensural bistable mechanisms (FTBM) accurately with fewer parameters.

From Figs. 5-8, it is evident that the tri-root bistable function (Eq. 8) represents the bistable kinetostatic behaviors easily and explicitly with fewer parameters for different kinds of compliant bistable mechanisms.

\section{Regression analysis of the classic fully-compliant bistable mechanism}

In this section, with the help of a mathematical optimization and analysis software called 1stOpt, we make the regression analysis for the classic fully-compliant bistable mechanism shown in Fig. 1 (its design relies heavily on NFEA because there is no appropriate model for this kind of bistable mechanism). The regression analysis can obtain expressions that explicitly express the relationships between the design parameters and the key performances of bistable designs in the form of polynomials.

As shown in Fig. 1, the classic fully-compliant bistable mechanism has six main design parameters $\left(L_{1}, L_{2}, L_{3}, \theta_{1}\right.$, $\theta_{2}$ and $\theta_{3}$ ). Given a group of design parameters, to model its bistable kinetostatic behaviors with the tri-root bistable function (Eq. 8), we have to find out the relationships among the five key points and design parameters. Here, we propose six closed-form equations to describe the relationships. Here $x_{2}$ is expressed as:

$x_{2}=a_{2} L_{1}^{2}+b_{2} L_{3}^{2}+c_{2} \theta_{1}^{3}+d_{2} \theta_{3}^{3}$

$+\sum_{i=0}^{2} \sum_{j=0}^{1} \sum_{k=0}^{2} \sum_{l=0}^{1} \sum_{m=0}^{1} \sum_{n=0}^{1} g_{i j k l m n} \theta_{1}^{i} \theta_{2}^{j} \theta_{3}^{k} L_{1}^{l} L_{2}^{m} L_{3}^{n}$

where $a_{2}, b_{2}, c_{2}, d_{2}$ and $g_{i j k l m n}$ are the unknown coefficients of all the terms in Eq. (19), which can be obtained by 1stOpt, which is introduced in Appendix A.

Similarly, the key points $x_{3}, x_{4}, x_{5}, F\left(x_{4}\right)$ and $F\left(x_{5}\right)$ can be given as

$$
\begin{aligned}
& x_{3}=a_{3} L_{1}^{3}+b_{3} L_{3}^{3}+c_{3} \theta_{1}^{2}+d_{3} \theta_{3}^{2} \\
& +\sum_{i=0}^{1} \sum_{j=0}^{1} \sum_{k=0}^{1} \sum_{l=0}^{2} \sum_{m=0}^{1} \sum_{n=0}^{2} h_{i j k l m n} \theta_{1}^{i} \theta_{2}^{j} \theta_{3}^{k} L_{1}^{l} L_{2}^{m} L_{3}^{n} \\
& x_{4}=a_{4} L_{1}^{4}+b_{4} L_{3}^{4}+c_{4} \theta_{1}^{2}+d_{4} \theta_{3}^{2} \\
& +\sum_{i=0}^{1} \sum_{j=0}^{1} \sum_{k=0}^{1} \sum_{l=0}^{3} \sum_{m=0}^{1} \sum_{n=0}^{3} f_{i j k l m n} \theta_{1}^{i} \theta_{2}^{j} \theta_{3}^{k} L_{1}^{l} L_{2}^{m} L_{3}^{n} \\
& x_{5}=a_{5} L_{1}^{4}+b_{5} L_{3}^{4}+c_{5} \theta_{1}^{2}+d_{5} \theta_{3}^{2} \\
& +\sum_{i=0}^{1} \sum_{j=0}^{1} \sum_{k=0}^{1} \sum_{l=0}^{3} \sum_{m=0}^{1} \sum_{n=0}^{3} p_{i j k l m n} \theta_{1}^{i} \theta_{2}^{j} \theta_{3}^{k} L_{1}^{l} L_{2}^{m} L_{3}^{n} \\
& F_{3}\left(x_{4}\right)=a_{40} L_{1}^{3}+b_{40} L_{3}^{3}+c_{40} \theta_{1}^{2}+d_{40} \theta_{3}^{2} \\
& +\sum_{i=0}^{1} \sum_{j=0}^{1} \sum_{k=0}^{1} \sum_{l=0}^{2} \sum_{m=0}^{1} \sum_{n=0}^{2} r_{i j k l m n} \theta_{1}^{i} \theta_{2}^{j} \theta_{3}^{k} L_{1}^{l} L_{2}^{m} L_{3}^{n} \\
& F_{3}\left(x_{5}\right)=a_{50} L_{1}^{3}+b_{50} L_{3}^{3}+c_{50} \theta_{1}^{3}+d_{50} \theta_{3}^{3} \\
& +\sum_{i=0}^{2} \sum_{j=0}^{1} \sum_{k=0}^{2} \sum_{l=0}^{2} \sum_{m=0}^{1} \sum_{n=0}^{2} q_{i j k l m n} \theta_{1}^{i} \theta_{2}^{j} \theta_{3}^{k} L_{1}^{l} L_{2}^{m} L_{3}^{n}
\end{aligned}
$$

respectively.

Four hundred fifteen classic fully-compliant bistable mechanism designs were analyzed by NFEA in order to achieve the five key points for nonlinear regression. The scopes of the geometric parameters of the designs are: $4 \mathrm{~mm} \leq L_{1} \leq 16 \mathrm{~mm}, 15 \mathrm{~mm} \leq L_{2} \leq 25 \mathrm{~mm}$, 


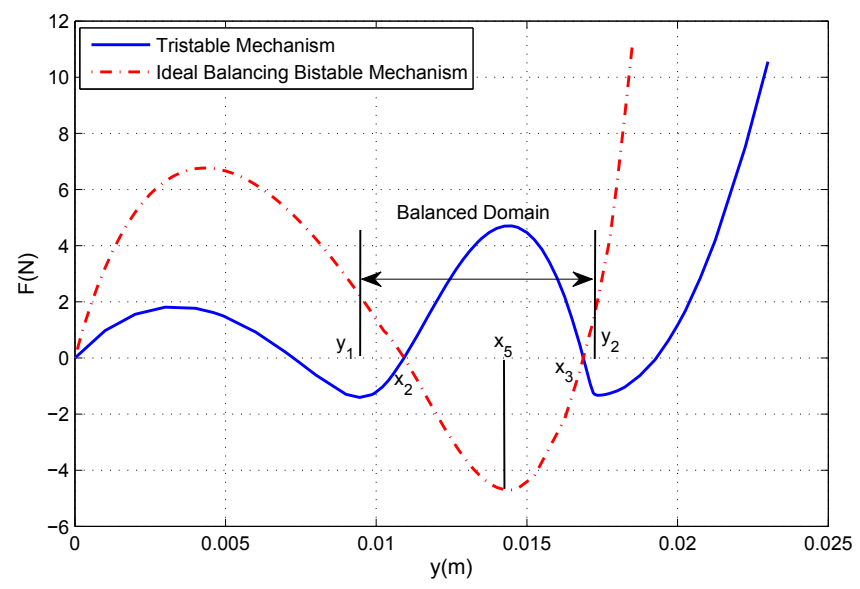

Figure 12. Force-displacement characteristics of the compliant tristable mechanism and a supposed ideal balancing bistable mechanism.

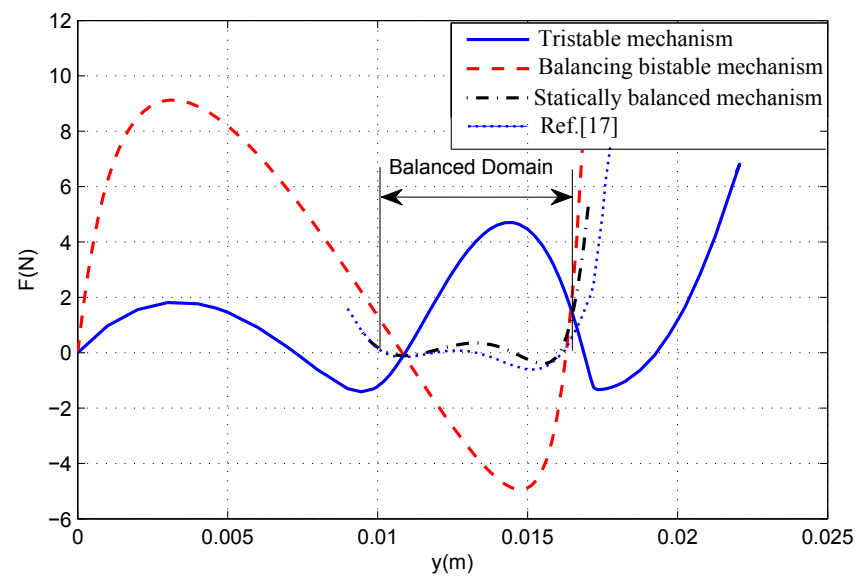

Figure 13. Force-displacement characteristics of the fullycompliant statically balanced mechanism.

$4 \mathrm{~mm} \leq L_{3} \leq 16 \mathrm{~mm}, 0 \leq \theta_{1} \leq 12^{\circ}, 7^{\circ} \leq \theta_{2} \leq 16^{\circ}$, and $0 \leq$ $\theta_{3} \leq 12^{\circ}$. The FEA model of each design was meshed with SOLID187 elements in ANSYS. A SOLID187 element is defined by ten nodes, each of which has three degrees of freedom. The model was meshed automatically by ANSYS. To ensure the accuracy of the FEA results, the mesh of each model was refined in the compliant segments so that no less than four elements were generated across the thinnest part, as shown in Fig. 9. Each model was fixed on the both ends of the compliant limb, loaded with a displacement on the shuttle, and the geometric nonlinearity option was turned on (Command "NLGEOM, ON"). When the solution was done, the corresponding force-displacement behaviors were recorded, which were then used to obtain the five key points.

Equations (19)-(24) were obtained by 1stOpt through fitting the FEA results of 415 designs. These equations explicitly express the relationships between the design parameters and the key performances of bistable designs in the form of

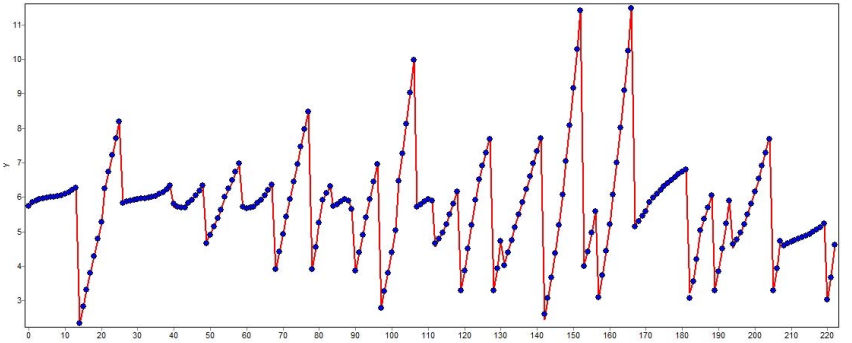

Figure 14. The fitting curve of Eq. (19) $\left(x_{2}\right)$.

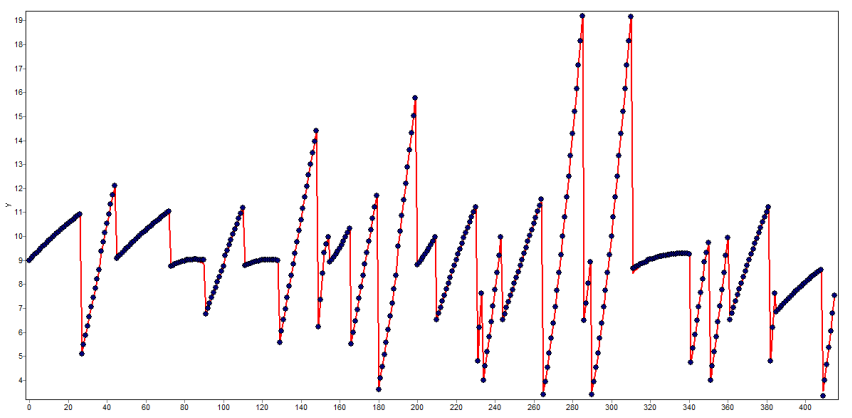

Figure 15. The fitting curve of Eq. (20) $\left(x_{3}\right)$.

polynomials, thus can serve as a mathematical model for this kind of bistable mechanism. The regression results are presented in Figs. 14-19 in Appendix B, and greatly simplify the design of future mechanisms of the classic fully-compliant bistable mechanism.

Regressing through the origin may ruin the accuracy of the fitting results (Eisenhauer, 2003). To avoid this problem, we chose $(-1,0)$ as the initial positions of the bistable curves in this paper. Once the kinetostatic behavior curve is determined, we translate the whole curve to the right of one unit (i.e. put the initial position of the curve at $(0,0)$ ).

Given the design parameters listed in Table 1, then the five key points of the bistable kinetostatic behavior curve can be calculated with the six closed-form equations (Eqs. 19-23). Thus the tri-root bistable function can be determined, which is plotted in Fig. 10 and expressed as:

$$
\begin{aligned}
& F_{3}(x)= \\
& \frac{-11.0208 x^{3}+129.1472 x^{2}-276.8704 x-417.0385}{x^{2}+5.4928 x-134.5362}
\end{aligned}
$$

where $x$ is the travel displacement of the shuttle from its initial equilibrium position. The total strain energy stored in the 


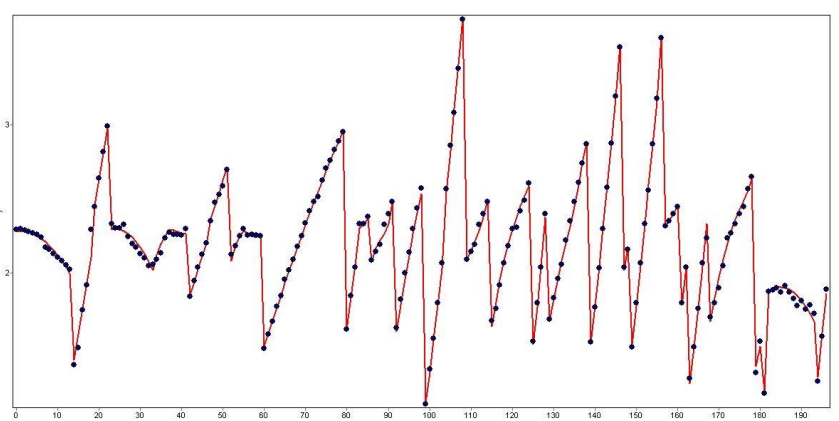

Figure 16. The fitting curve of Eq. (21) $\left(x_{4}\right)$.

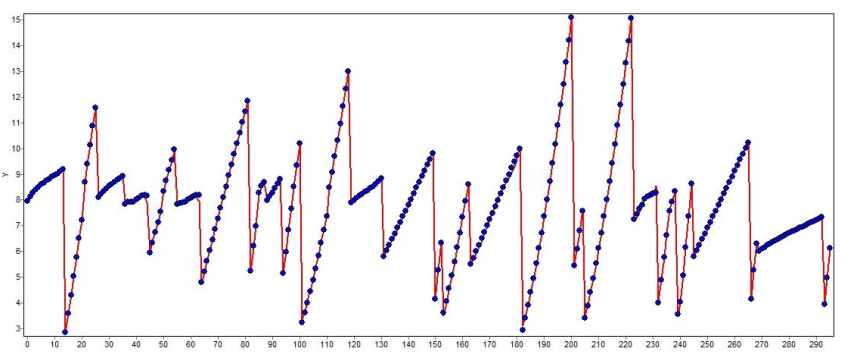

Figure 17. The fitting curve of Eq. (22) $\left(x_{5}\right)$.

whole bistable mechanism at any position is given as:

$$
\begin{aligned}
& V(x)= \\
& \int_{-1}^{x} \frac{-11.0208 x^{3}+129.1472 x^{2}-276.8704 x-417.0385}{x^{2}+5.4928 x-134.5362} \\
& \mathrm{~d} x-1 \leq x \leq 7.985
\end{aligned}
$$

where, $x=7.985$ is the second stable equilibrium position. The curve of strain energy is plotted compared with nonlinear finite element analysis (NFEA) in Fig. 10. It is obvious that the errors are very little and the tri-root bistable function can mathematically model the classic fullycompliant bistable mechanism accurately. This may greatly simplify the modeling of future mechanisms of this type.

\section{Design of a fully-compliant statically-balanced mechanism}

In this section, to validate the regression results, we present a fully-compliant statically-balanced mechanism combining three different classic fully-compliant bistable mechanisms that can ideally provide zero stiffness free motion. This mechanism has been proposed and studied by Chen and Zhang (2011).

As shown in Fig. 11, the fully-compliant staticallybalanced mechanism is designed with a specific tristable mechanism (a) and a balancing bistable mechanism (b): the tristable mechanism consists of two different compliant

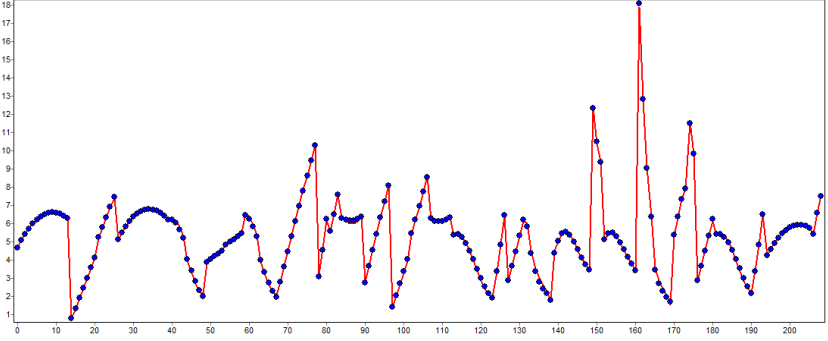

Figure 18. The fitting curve of Eq. (23) $\left(F\left(x_{4}\right)\right)$.

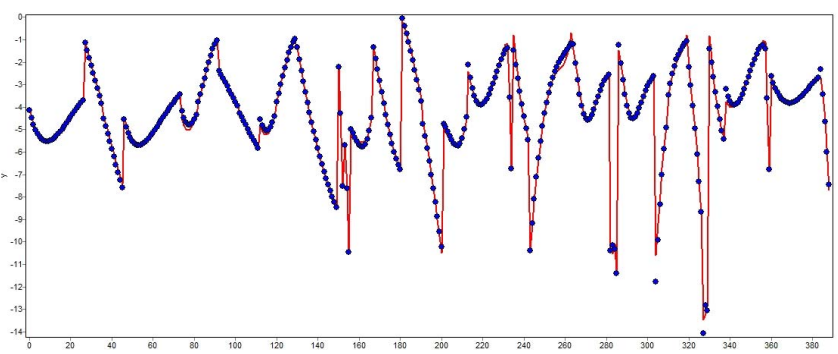

Figure 19. The fitting curve of Eq. (24) $\left(F\left(x_{5}\right)\right)$.

bistable mechanisms (i.e., Part A and Part B) connected in series, as shown in Fig. 11(a). Its design parameters are shown in Fig. 1 and listed in Table 4. The force-displacement characteristics of the tristable mechanism are achieved using nonlinear finite element analysis (NFEA) and plotted in Fig. 12. To statically balance the whole mechanism, Chen and Zhang (2011) proposed a method that: first supposes there exit an ideal balancing bistable mechanism which can counterbalance the given tristable mechanism. Then we can obtain the force-displacement characteristics of the supposed bistable mechanism and the approximate statically-balanced domain (from $y_{1}$ to $y_{2}$, shown in Fig. 12). Finally, a group of ideal design parameters for the balancing bistable mechanism is found using a particle swarm optimizer (PSO) (Chen et al., 2011a) integrated with ANSYS. Reference Chen and Zhang (2011) gives a detailed description for this process.

To avoid the optimization process, we propose a straightforward method combining the tri-root bistable function and regression analysis results introduced above: First we suppose an ideal balancing bistable mechanism and obtain its force-displacement characteristics as shown in Fig. 12, then we get the five key points $x_{1}=0, x_{2}=10.93 \mathrm{~mm}, x_{3}=$ $16.87 \mathrm{~mm}, x_{5}=14.5 \mathrm{~mm}$ and $F\left(x_{5}\right)=-4.7$. We assume $H=6 \mathrm{~mm}, \theta_{1}=\theta_{3}=0, w_{1}=w_{3}=0.8 \mathrm{~mm}$ and $w_{2}=3 \mathrm{~mm}$ for the balancing bistable mechanism, thus the unknown design parameters we want are reduced to $4\left(L_{1}, L_{2}, L_{3}\right.$ and $\theta_{2}$ ). By solving the four closed-form equations (Eqs. 19, 20, 22 and 24) simultaneously, the four design parameters can be determined, which are listed in Table 4.

The force-displacement characteristics of the tristable mechanism, balancing bistable mechanism and the balanced 
domain of the whole statically-balanced mechanism are plotted in Fig. 13.

Figure 13 shows that the whole mechanism has approximately balanced behavior during $y_{1}$ and $y_{2}$. Compared with the optimized results obtained by Chen and Zhang (2011), the statically-balanced behaviors achieved in this paper are accurate and convenient. It indicates that this method can substitute the optimization process in some respects. Meantime, it also validates the high accuracy of the regression analysis made above.

\section{Conclusions}

In this paper, we proposed a rational function called "triroot bistable function" to describe the complete bistable kinetostatic behaviors briefly and accurately with few parameters. The tri-root bistable function has been shown to have a high precision by three different kinds of compliant bistable mechanisms. Then, six closed-form equations capturing the relationships among the five key points and design parameters were proposed and obtained by a multivariable nonlinear regression analysis for the classic fullycompliant bistable mechanism. This process was achieved by 1stOpt. Finally, a fully-compliant statically-balanced mechanism consisting of three different classic fully-compliant bistable mechanisms was presented and designed with the proposed method to show the capability of the tri-root bistable function in design and analysis of bistable mechanisms.

It should be noted that, the tri-root bistable function and regression analysis can also be employed in other types compliant bistable mechanisms (such as the Young bistable mechanism and FTBM illustrated in this paper) for modeling and design. 


\section{Appendix A}

1stOpt (First Optimization) is a set of integrated tools for mathematical analysis and optimization software package. It is adept at nonlinear regression, curve fitting and other nonlinear project problems. The nuclear arithmetic is Universal Global Optimization (UGO). Its specialty is that, the initial values of all the parameters are offered by 1stOpt randomly instead of the users, as it is a difficulty for most of the people. Even though, the globally-optimal solution can be found rapidly and precisely.

In this paper, one of the difficulties is how to do the multivariable nonlinear regression rapidly and precisely, which are used to determine the parameters in the six closedform equations (Eqs. 19-24). In 1stOpt, there are about four methods can do this, Liveners-Marquetry (LM), QuasiNewton (BFGS), Simplex Method (SM) and Differential Evolution (DE), they are all associated with UGO to obtain the globally-optimal solution. As each method has its own merits and drawbacks, we proposed to do the regression combining all the four methods, which was proved precisely and efficiently.

\section{Appendix B}

The fitting curves of the six closed-form equations (Eqs. 1924), which are obtained by 1 stOpt and shown as following (Figs. 14-19). 
Acknowledgements. The authors gratefully acknowledge the financial support from the National Natural Science Foundation of China under Grant No. 51175396, the Specialized Research Fund for the Doctoral Program of Higher Education of China under Grant No. 20120203110015, and the Fundamental Research Funds for the Central Universities under No. K5051204021.

Edited by: G. Hao Reviewed by: L. Howell and one anonymous referee

\section{References}

Andò, B., Baglio, S., L'Episcopo, G., and Trigona, C.: Investigation on mechanically bistable MEMS devices for energy harvesting from vibration, J. Microelectromech. Syst., 21(4): 779-790, 2012.

Charlot, B., Sun, W., Yamashita, K., Fujita, H., and Toshiyoshi, H.: Bistable nanowire for micromechanical memory, J. Micromech. Microeng, 18, 045005, doi:10.1088/0960-1317/18/4/045005, 2008.

Chen, G. and Du, Y.: Double-Young tristable mechanisms, ASME J. Mechan. Robot., 5, 011007, doi: 10.1115/1.4007941, 2013.

Chen, G. and Zhang, S.: Fully-compliant statically-balanced mechanisms without prestressing assembly: concepts and case studies, Mechan. Sci., 2, 169-174, 2011.

Chen, G., Aten, Q. T., Zirbel, S., Jensen, B. D., and Howell, L. L.: A tristable mechanism configuration employing orthogonal compliant mechanisms, ASME J. Mechan. Robot., 2, 014501, doi:10.1115/1.4000529, 2009a.

Chen, G., Wilcox, D. L., and Howell, L. L.: Fully compliant double tensural tristable micromechanisms (DTTM), J. Micromech. Microeng, 19, 025011, doi:10.1088/0960-1317/19/2/025011, 2009b.

Chen, G., Xiong, B., and Huang, X.: Finding the optimal characteristic parameters for 3R pseudo-rigid-body model using an improved particle swarm optimizer, Precis. Eng. , 35, 505-511, 2011a.

Chen, G., Gou, Y., and Zhang, A.: Synthesis of compliant multistable mechanisms through use of a single bistable mechanism, ASME J. Mechan. Design, 133, 081007, doi:10.1115/1.4004543, $2011 b$.

Eisenhauer, J. G.: Regression through the origin, Teaching Statist., 25, 76-80, 2003.

Hafez, M., Lichter, M. D., and Dubowsky, S.: Optimized binary modular reconfigurable robotic devices, IEEE/ASME TRANS. Mech., 8, 304-326, 2003.

Halverson, P. A., Howell, L. L., and Magleby, S. P.: Tension-based multi-stable compliant rolling-contact elements, Mech. Mach. Theory, 45, 147-156, 2010.

Hansen, B. J., Carron, C. J., Jensen, B. D., Hawkins, A. R., and Schultz, S. M.: Plastic latching accelerometer based on bistable compliant mechanisms, Smart Mater. Struct., 16, 1967-1972, 2007.
Hoffmann, M., Kopka, P., and Voges, E.: Bistable micromechanical fiber-optic switches on silicon with thermal actuators, Sens. Actuators: A, 78, 28-35, 1999.

Howell, L. L.: Compliant Mechanisms, Wiley, New York, 2001.

Jensen, B. D. and Howell, L. L.: Identification of compliant pseudorigid-body mechanism configurations resulting in bistable behavior, Trans. ASME, J. Mechan. Design, 125, 701-708, 2003.

Jensen, B. D. and Howell, L. L.: "Bistable configurations of compliant mechanisms modeled using four links and translational joints, Trans. ASME, J. Mechan. Design, 126, 657-666, 2004.

Jensen, B. D., Howell, L. L., and Salmon L. G.: Design of two-link, in-plane, bistable compliant micro-mechanisms, Trans. ASME, J. Mechan. Design, 121, 416-423, 1999.

Jensen, B. D., Parkinson, M. B., Kurabayashi, K., Howell, L. L., and Baker, M. S.: Design optimization of a fully-compliant bistable micro-mechanism, Proc. ASME IMECE (New York, USA, 1116 Nov. 2001), 2, 2931-2937, 2001.

Masters, N. D. and Howell, L. L.: A self-retracting fully compliant bistable micromechanism, J. Microelectromech. Syst., 12, 273 280, 2003.

Oberhammer, J., Tang, M., Liu, A. Q., and Stemme, G.: Mechanically tri-stable, true single-pole-double-throw (SPDT) switches, J. Micromech. Microeng., 16, 2251-2258, 2006.

Oh, Y. S. and Kota, S.: Synthesis of multistable equilibrium compliant mechanisms using combinations of bistable mechanisms, Trans. ASME, J. Mechan. Design, 131, 021002, doi:10.1115/1.3013316, 2009.

Pendleton, T. M. and Jensen, B. D.: Development of a tristable compliant mechanism, Proc. 12TH IFToMM World Congress, A835, 2007.

Pucheta, M. A. and Cardona, A.: Design of bistable compliant mechanisms using precision-position and rigid-body replacement methods, Mech. Mach. Theory, 45, 304-326, 2010.

Sönmez, Ü. and Tutum, C. C.: A compliant bistable mechanism design incorporating elastica buckling beam theory and pseudo-rigid-body model, Trans. ASME, J. Mechan. Design, 130, 042304, doi:10.1115/1.2839009, 2008.

$\mathrm{Su}, \mathrm{H}$. and McCarthy, J. M.: Synthesis of bistable compliant fourbar mechanisms using polynomial homotopy, Trans. ASME, J. Mechan. Design, 129, 1094-1098, 2007.

Tolou, M., Estevez, P., and Herder, J. L.: Collinear-type statically balanced compliant micro mechanism (SB-CMM): experimental comparison between pre-curved and straight beams, Proceedings of the ASME Design Engineering Technical Conferences \& Computers and Information in Engineering Conference, 28-31 August 2011, Washington, DC, USA, DETC2011-47678, 2011.

Wang, D.-A., Phama, H.-T., and Hsieh, Y.-H.: Dynamical switching of an electromagnetically driven compliant bistable mechanism, Sensors and Actuators A: Physical, 43, 143-151, 2009.

Wilcox, D. L. and Howell, L. L.: Fully compliant tensural bistable micromechanisms (FTBM), J. Microelectromech. Syst., 14, 1223-1235, 2005. 